\title{
Performance and Aging Studies of BaBar Resistive Plate Chambers
}

H. R. Band ${ }^{*} \dagger$, J. Hollar ${ }^{\mathrm{a}}$, P. Tan ${ }^{\mathrm{a}}$, F. Anulli ${ }^{\mathrm{b}}$, R. Baldinib ${ }^{\mathrm{b}}$, A. Calcaterra ${ }^{\mathrm{b}}$, R. de Sangro ${ }^{\mathrm{b}}$, G.

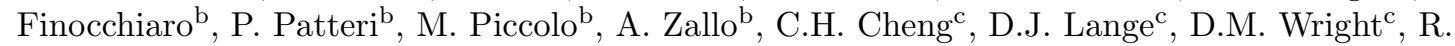
Messner $^{\mathrm{d}}$, W.J. Wisniewskit ${ }^{\mathrm{d}}$, M. Pappagallo ${ }^{\mathrm{e}}$, M. Andreotti ${ }^{\mathrm{f}}$, D. Bettonif ${ }^{\mathrm{f}}$, R. Calabrese $^{\mathrm{f}}$, G.

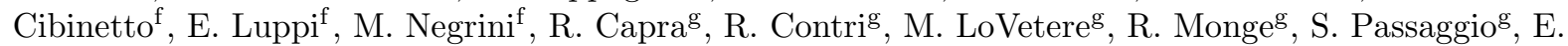

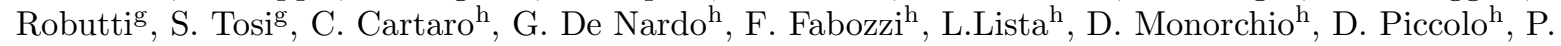
Paolucci $^{\text {h}}$, R. Covarelli ${ }^{i}$, M. Pioppi ${ }^{i}$, M. Carpinelli ${ }^{j}$, F. Forti $^{j}$, N. Neri ${ }^{j}$, E. Paoloni ${ }^{j}$, F. Bellini ${ }^{k}$, G. Cavoto $^{k}$, E. Di Marcok ${ }^{k}$ A. D'Orazio ${ }^{\mathrm{k}}$, D. del Re ${ }^{\mathrm{k}}$, R. Faccini ${ }^{\mathrm{k}}$, F. Ferrarotto ${ }^{\mathrm{k}}$, F. Ferronik ${ }^{\mathrm{k}}$, M. Gaspero $^{\mathrm{k}}$, L. Li Gioi ${ }^{\mathrm{k}}$, M.A. Mazzoni ${ }^{\mathrm{k}}$, S. Morganti ${ }^{\mathrm{k}}$, M. Pierini ${ }^{\mathrm{k}}$, G. Pireddak ${ }^{\mathrm{k}}$, C. Voena ${ }^{\mathrm{k}}$, C. Potter ${ }^{\mathrm{l}}$,

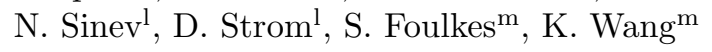

${ }^{a}$ University of Wisconsin, Madison, WI, USA

${ }^{\mathrm{b}}$ Laboratori Nazionali di Frascati, INFN, Italy

${ }^{\mathrm{c}}$ Lawrence Livermore National Laboratory, Livermore, CA, USA

${ }^{\mathrm{d}}$ Stanford Linear Accelerator Center, Menlo Park, CA, USA

${ }^{\mathrm{e}}$ Università di Bari \& INFN Sezione di Bari, Italy

${ }^{\mathrm{f}}$ Università di Ferrara \& INFN Sezione di Ferrara, Italy

gUniversità di Genova \& INFN Sezione di Genova, Italy

${ }^{\text {h} U n i v e r s i t a ̀ ~ d i ~ N a p o l i ~ " F e d e r i c o ~ I I " ~ \& ~ I N F N ~ S e z i o n e ~ d i ~ N a p o l i, ~ I t a l y ~}$

${ }^{\mathrm{i} U n i v e r s i t a ̀ ~ d i ~ P e r u g i a ~ \& ~ I N F N ~ S e z i o n e ~ d i ~ P e r u g i a, ~ I t a l y ~}$

${ }^{j}$ Università di Pisa \& INFN Sezione di Pisa, Italy

"Università di Roma "La Sapienza" \& INFN Sezione di Roma, Italy

${ }^{1}$ University of Oregon, Eugene, OR, USA

${ }^{\mathrm{m}}$ University of California, Riverside, CA, USA

The BaBar detector is currently operating nearly 200 Resistive Plate Chambers (RPCs), constructed as part of an upgrade of the forward endcap muon detector in 2002. Although the average RPC efficiency remains high, numerous changes in the RPC performance (increased currents and rates) have been observed. A few of the highest rate RPCs have suffered efficiency losses of more than 15\%. Several types of efficiency loss have been observed. Tests with humidified gas have shown that some of the lost efficiency is recoverable. However, efficiency losses in the highest rate regions have not yet improved with humid gases.

\section{Introduction}

The BaBar detector collaboration [1] installed

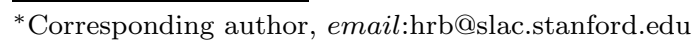
$\dagger^{\dagger}$ This work was supported by U.S. Dept. of Energy grant DE-FG02-95ER40896 over 200 2nd generation Resistive Plate Chambers [2] (RPCs) as part of an upgrade [3] of the 
forward endcap muon and neutral hadron detector (IFR) in 2002 and continues to operate over 400 original production RPCs in the barrel and backward endcap. BaBar RPCs are constructed from Bakelite treated with linseed oil and operate in limited streamer mode, using a gas mixture of $4.5 \%$ isobutane, $60.6 \%$ argon and Freon 134a.

Unlike the original production of RPCs for BaBar, the new RPCs have performed reliably, however, clear signs of aging and efficiency loss are seen in the higher rate chambers [4]. In this note we describe the latest data on currents and efficiencies and present a new study of the effect of gas humidity on the RPC efficiency.

\section{Instrumented Flux Return}

BaBar RPCs reside in gaps of the barrel and endcap flux return steel as seen in Fig. 1. The streamer rates produced by backgrounds and signals from normal BaBar running vary considerably depending on the layer and position of the chambers, thus providing a broad spectrum test

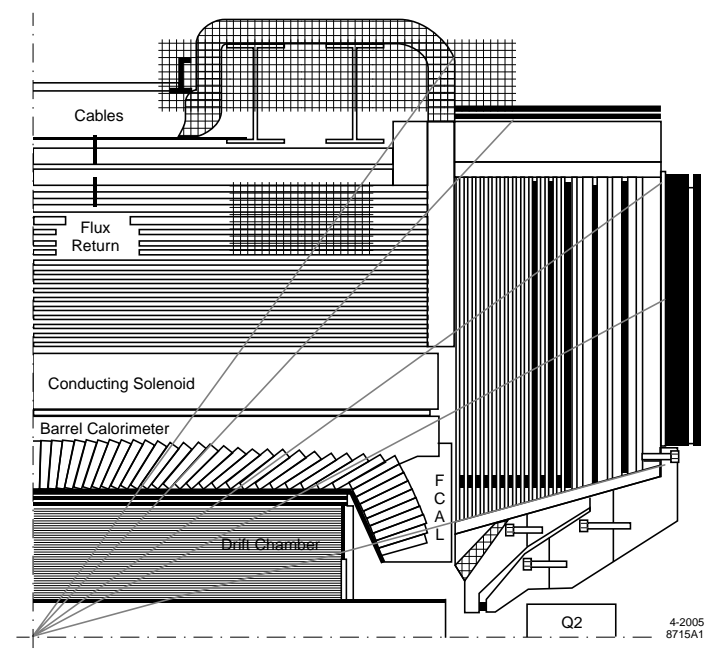

Figure 1. A longitudinal section of the BaBar detector in 2005 showing the details of the flux return steel surrounding the inner detector elements. Planar RPCs fill the gaps in the steel in the barrel and endcaps. The new endcap contains 16 RPC planes numbered from left to right.

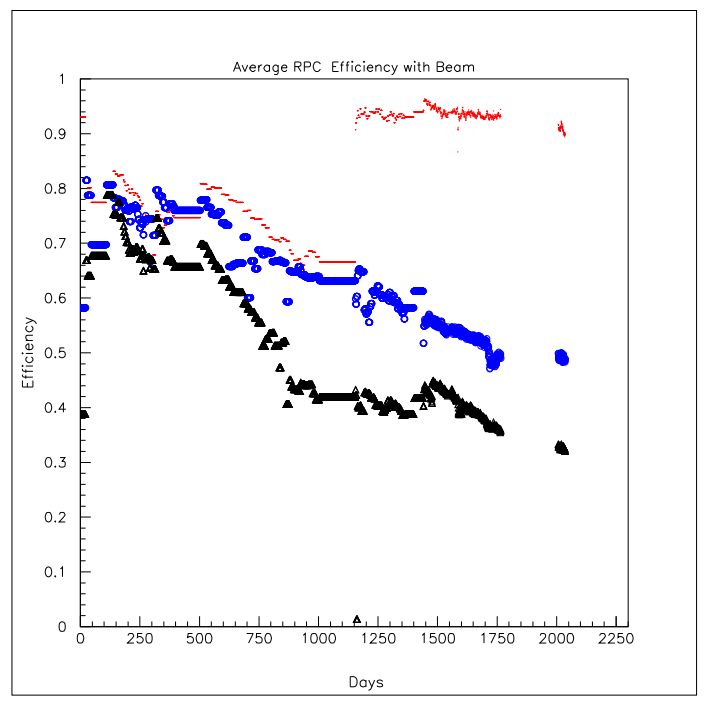

Figure 2. The average RPC efficiency determined from $\mu$ pairs since November, 1999 for the barrel(triangles), forward endcap(points) and backward endcap(open circles).

of RPC performance and aging. Signal rates, currents, and occupancy are generally proportional to PEPII luminosity with peak rates above 15 $\mathrm{Hz} \mathrm{cm}^{2}$ in the regions closest to the beampipe in the forward endcap. The rates in the outermost layers (13-16) were sensitive to backgrounds from PEPII entering the outside of the endcap and were typically $2-4 \mathrm{~Hz} \mathrm{~cm}{ }^{2}$.

The average RPC efficiencies per chamber were measured by $\mu$ pairs during colliding beam data and periodically by cosmic ray runs. The average efficiency from $\mu$ pairs since the start of BaBar data-taking in November, 1999 is shown Fig. 2. Unlike the barrel and backward endcap, efficiency of the new forward endcap has been stable, but has declined in the most recent data. The efficiency determined from cosmic rays, however, has stayed high, suggesting the efficiency losses are rate dependent.

The effect of the rate on the long term behavior of the RPC efficiency is shown in Fig. 3 


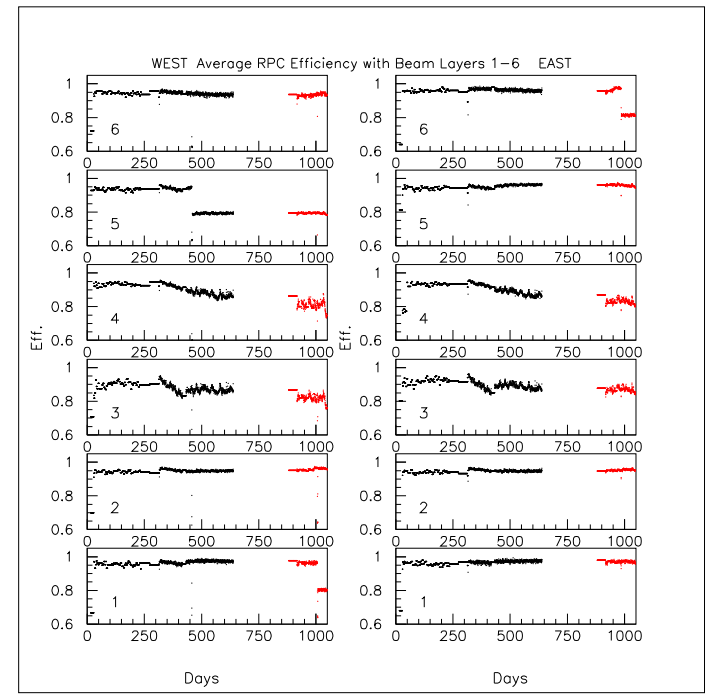

Figure 3. The average RPC module efficiency of layers 1-6 determined from $\mu$ pairs. The modules in each door are numbered from 1 to 6 starting from the floor. Positions 3 and 4 are closest to the beamline and have the highest background rates.

where the average efficiency of the first 6 layers of the East and West doors are plotted versus time. The modules, which are numbered 1-6 starting at the floor, show different behavior. The chambers with the lower rates $(1,2,4$ and 6$)$ are very stable. The only changes are due to 3 RPCs developing HV problems. The RPCs in positions 3 and 4 exhibit an efficiency loss. The rate of decline of the RPCs in position 3 was slowed by the reversal of direction of the gas flow(RPCs 3 and 4 are in series) on day 420. A study of the efficiency as a function of position shows that the decline is in the high rate regions near the beamline.

\section{Gas Humidity Tests}

New studies have investigated the effect of gas humidity on the RPC efficiency as a function of rate. The relative humidity of the IFR input gas was near $0 \%$, while the exhaust gas from 2 RPCs in series is typically $20-30 \%$, thus presum- ably removing water from the chambers. Inefficient regions developed in many RPCs near the gas inlets. These regions were small in the inner layers where the noise rate is low far from the beamline and much larger in the outer layers where the background rates were uniformly high. Since these regions were efficient without beam, it seemed likely that the inefficiency was caused by the drying of the Bakelite which had increased the Bakelite bulk resistance as seen in Atlas RPC studies [5]. The efficiency of the outermost endcap layer which was most sensitive to the Bakelite resistivity (because of the high noise rate) is shown in Fig. 4 before the introduction of humidified gas. This layer had been off for most of the running before 2005 and displayed no other signs of aging. The gas was then humidified to about $30 \% \mathrm{RH}$ by bubbling part of the gas through water. The efficiency of the RPCs slowly improved to near nominal as seen in Fig. 5 which was taken nearly 5 months after the introduction of the humid gas. No significant changes in the moisture level of the gas coming out of these RPCs was observed.

\section{Small Radii Efficiency}

As described earlier the efficiency decline observed in the RPCs near the beam-line was correlated with the typical noise rate in those RPCs. To study this effect the efficiency was plotted as a function of the radius from the beam-line. The efficiency at the peak of the background distribution was seen to decrease steadily from the start of running in the fall of $2002(90 \%)$ to the present(less than $60 \%$ ) in layer 1 . The efficiency at small radii of layers in the middle of the forward endcap with lower noise rates decreased by smaller amounts and at later times compared to layer 1. Efficiencies at larger radii have been stable at greater than $93 \%$. The humid input gases have made no change to the above trends to date.

\section{Conclusions}

The operational parameters of the 2nd generation BaBar RPCs continue to evolve as the chambers age. The overall performance of the 


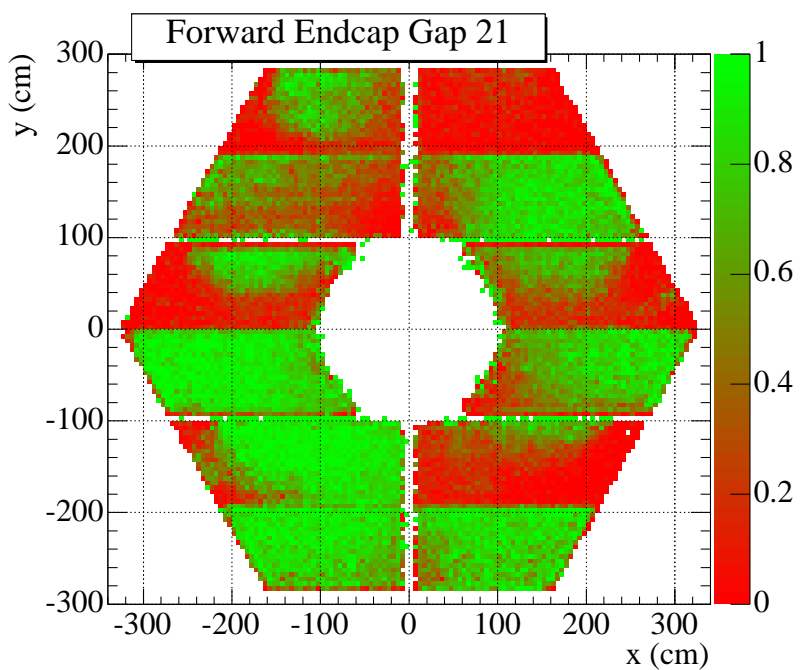

Figure 4. The two dimensional RPC efficiency of layer 16 measured with $\mu$-pairs in April 2005 after 30 months of dry gas flow.

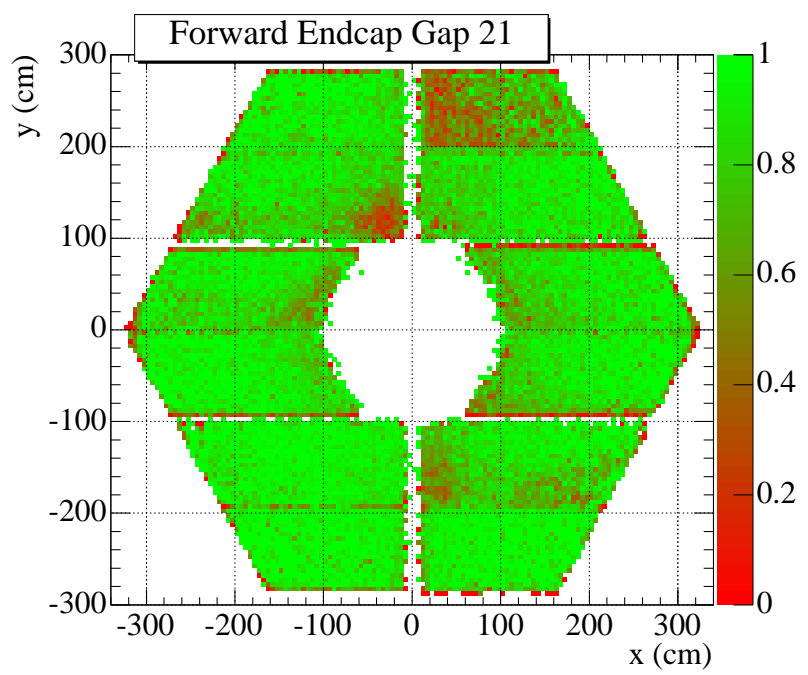

Figure 5. The two dimensional RPC efficiency of layer 16 measured with $\mu$-pairs in the Sep. 05 after 5 months of gas flow humidified to about $30 \% \mathrm{RH}$. new chambers is much superior to the original BaBar RPCs, justifying the design changes and care of construction. However, clear aging effects are seen in the highest rate regions. Control of the input gas humidity has been established as an important parameter to rehumidify dried Bakelite surfaces and thus restore the rate capability of the chambers.However humid gas has not been effective in restoring the efficiency in the very highest rate regions. RPCs with noise and background rates below $2-4 \mathrm{~Hz}$ per $\mathrm{cm}^{2}$ have proven to be very stable in both rates, currents and efficiency. Further study is planned to quantify the relationship between the integrated charge accumulated in each RPC region and the observed efficiency changes.

\section{ACKNOWLEDGMENTS}

We wish to thanks our PEPII colleagues for their dedicated efforts to constantly improve luminosity and reduce detector backgrounds. This work was supported by the U.S. Dept. of Energy and the I.N.F.N in Italy.

\section{REFERENCES}

1. B. Aubert et al., Nucl. Instr. and Meth. A 479 (2002) 1.

2. R. Santonico and R. Cardarelli, Nucl. Inst and Meth. A 187 (1981) 377.

3. F. Anulli, et al., Nucl. Instr. and Meth. A 539 (2005) 155-171.

4. F. Anulli, et al., Nucl. Instr. Meth. A 552(2005) 276-291.

5. G. Aielli, et al., Nucl. Instr. Meth. A 533(2004) 86-92. 